\title{
DOS NUEVAS ESTELAS FUNERARIAS EN FRESNO DE RÍO TIRÓN (BURGOS)
}

\author{
TWO NEW FUNERARY STELES \\ AT FRESNO DE RÍO TIRÓN (BURGOS) \\ Marta Fernández Corral* y Bruno P. Carcedo de Andrés \\ $U P V / E H U-U B U$ \\ mfernandezcorral@gmail.com \\ bpcarcedo@ubu.es
}

DOI: $10.1387 /$ veleia.14989

\begin{abstract}
Resumen: El objetivo de este trabajo es dar a conocer dos nuevas estelas funerarias de época romana localizadas en el verano de 2014 y primavera de 2015 en Fresno de Río Tirón (Burgos). Las características de las estelas nos hacen pensar que son dos ejemplos más del llamado conjunto epigráfico de Belorado (Burgos) bien reconocibles debido a su soporte y características internas.

Palabras clave: epigrafía romana funeraria, Fresno de Río Tirón, conjunto epigráfico de Belorado.

Abstract: This paper presents the study of two new funerary steles from Roman period found in Fresno de Río Tirón (Burgos) in the summer of 2014 and the spring of 2015. The features of the steles suggest that they should be understood as new examples of the epigraphic group of Belorado (Burgos) where most of the steles share the same kind of support and some specific internal characteristics.

Keywords: Roman funerary epigraphy, Fresno de Río Tirón, epigraphic group of Belorado.
\end{abstract}

Recibido: 27-05-2015

Informado: 29-06-2015

Definitivo: 06-07-2015

\section{INTRODUCCIÓN $^{1}$}

A continuación presentamos dos nuevas inscripciones funerarias localizadas en Fresno de Río Tirón (Burgos) que, debido a sus características externas e internas, creemos que deben ser entendidas dentro del llamado conjunto de Belorado ${ }^{2}$. Este grupo de estelas, que ascienden a más de

\footnotetext{
* Programa de Formación de Personal Investigador No Doctor del Gobierno Vasco y Grupo de investigación del sistema universitario vasco IT-760-13.

${ }^{1}$ Nos gustaría comenzar agradeciendo a los editores de la revista y a los pares ciegos que han evaluado el tra-
}

bajo sus correcciones y comentarios para mejorar la calidad del texto final.

2 El estudio más completo y actualizado hasta la fecha de este conjunto es el realizado por Reyes Hernando (2000). 
40 ejemplares, guarda una serie de características comunes de las cuales, la más evidente, es la utilización como soporte de bloques fluviales ${ }^{3}$ de cuarcita sin trabajar ni decorar ${ }^{4}$. Debido a las características del soporte, la técnica utilizada es el repiqueteado en casi todos los casos. Por otro lado, la mayoría de las estelas, aunque no todas, presentan un texto encabezado por el nombre del difunto al que le sigue su filiación y la edad en la que falleció. Destaca igualmente la falta de fórmulas típicas de este tipo de inscripciones ${ }^{5}$ y la presencia de algunos signos habitualmente situados en la parte superior de la inscripción, de difícil interpretación ${ }^{6}$.

La aparición de estos monumentos funerarios ha sido relacionada con el yacimiento de La Mesa, situado en una pequeña meseta al oeste de Belorado donde se han encontrado abundantes muestras de cultura material de cronología romana y donde durante el 2014 se llevaron a cabo sondeos que dieron como resultado la identificación de una primera fase alto-imperial (siglos I-II d.C.) y una segunda fase más complicada de datar debido al arrasamiento del yacimiento pero, probablemente, vinculada a un momento más tardío del periodo imperial ${ }^{7}$.

\section{ANÁLISIS DE LAS ESTELAS}

1. (Fig. 1) El primero de los hallazgos fue realizado en junio de 2014 por Arturo Carcedo García en el cauce del río Tirón a su paso por este municipio, a 100 metros del puente aproximadamente. Según su descubridor, pudo ser arrastrado río abajo hasta el lugar donde fue localizado por las fuertes lluvias que hubo los días anteriores y que provocaron la crecida del río. A día de hoy, la pieza obra en poder del autor del hallazgo ${ }^{8}$.

La estela funeraria es, como ya hemos adelantado, un bloque fluvial de cuarcita de forma prismática que mide [60] $\times[27] \times 36 \mathrm{~cm}$. El bloque se encuentra fracturado en ambos laterales y en su parte superior. La fractura del lado derecho no parece haber afectado al campo epigráfico, sin embargo, la de la izquierda ha provocado que se hayan perdido la letra inicial de cada línea del texto. En el extremo superior del bloque se aprecian restos de repiqueteado de difícil identificación debido a la rotura de la pieza. El resto de texto conservado no presenta problemas de lectura y, como es habitual en las inscripciones de este lugar, no se observa ningún motivo decorativo.

El campo epigráfico se sitúa en la parte superior de la pieza, sin embargo, las fracturas del bloque nos impiden saber si se guardó margen a los lados. Resulta difícil saber, igualmente, si los restos de repicado que se aprecian en la parte superior son parte de una primera línea del texto o, tal

3 Su paralelo más cercano en cuanto a soporte sería el conjunto de estelas vadinienses (González Rodríguez 1997) a las que habría que añadir otras de Asturias, Soria y fuera de Hispania, en la IX Región de Italia (González Rodríguez 2002, 95).

4 La única excepción sería la estela discoidea realizada sobre un soporte de caliza y abundantemente decorada con motivos geométricos (Reyes Hernando 2000, n. $\left.{ }^{\circ} 29\right)$.

5 A excepción de la estela dedicada a Quemia Maglaena (Reyes Hernando 2000, n. ${ }^{\circ}$ 1) en la que encontramos la fórmula HSE.

6 Contamos con estas marcas en las n. ${ }^{\circ}$ 1, 3, 4, 7 y 9 de la publicación de Reyes Hernando 2000 y posible- mente también en las n. ${ }^{\circ} 11$ y 20 , así como en la n. ${ }^{\circ} 2$ y 4 de Fernández Corral 2015 y en las n.o 1 , 4 y 5 de Gorrochategui y Fernández Corral, e.p.

7 El yacimiento ya era conocido por los abundantes materiales de época romana localizados en superficie y estaba incluido en la Carta Arqueológica de Burgos (Abásolo, 1974, 11). Agradecemos a los arqueólogos responsables del proyecto Joan Oller Guzmán, Diana de León Subías y Delia Eguiluz Maestro la información proporcionada al respecto (Oller, De León, Eguiluz, 2015).

8 Agradecemos a Arturo Carcedo la información que nos ha facilitado sobre su hallazgo y permitirnos su estudio. 
vez, de un signo situado sobre el campo epigráfico, como ya hemos dicho, habituales entre las estelas de este lugar. Trataremos este problema más adelante.

En cualquier caso, el interlineado entre estos restos de repiqueteado (línea 1) y la primera línea identificable es de $1,7 \mathrm{~cm}$ mientras que el siguiente interlineado mide $0,5 \mathrm{~cm}$ y el último, 0,3-0,4 cm. En la línea central se aprecia una interpunción redonda y el nexo «AV». Las medidas de las letras crecen a medida que avanza el texto midiendo entre 4,5-5 cm en la segunda línea, 5-5,5 $\mathrm{cm}$ en la tercera y $6 \mathrm{~cm}$ en la última. El trazado es, por lo tanto, algo irregular y en las ocasiones en las que las letras se repiten, no son del todo similares. Este es el caso de la $\mathrm{R}$ que en la segunda línea aparece dibujada con la cola partiendo de la unión del seno y el asta mientras que en la tercera línea lo hace desde el seno o la letra $\mathrm{N}$ que en la segunda línea es más estilizada que en la última.

De igual modo, la distancia entre las letras también es irregular en la línea 2: entre I y R $1 \mathrm{~cm}$; entre $\mathrm{R}$ y $\mathrm{O} 1,3 \mathrm{~cm}$; entre $\mathrm{O}$ y N $1 \mathrm{~cm}$ y finalmente, una distancia significativa de $2,8 \mathrm{~cm}$ entre las letras $\mathrm{N}$ e I.

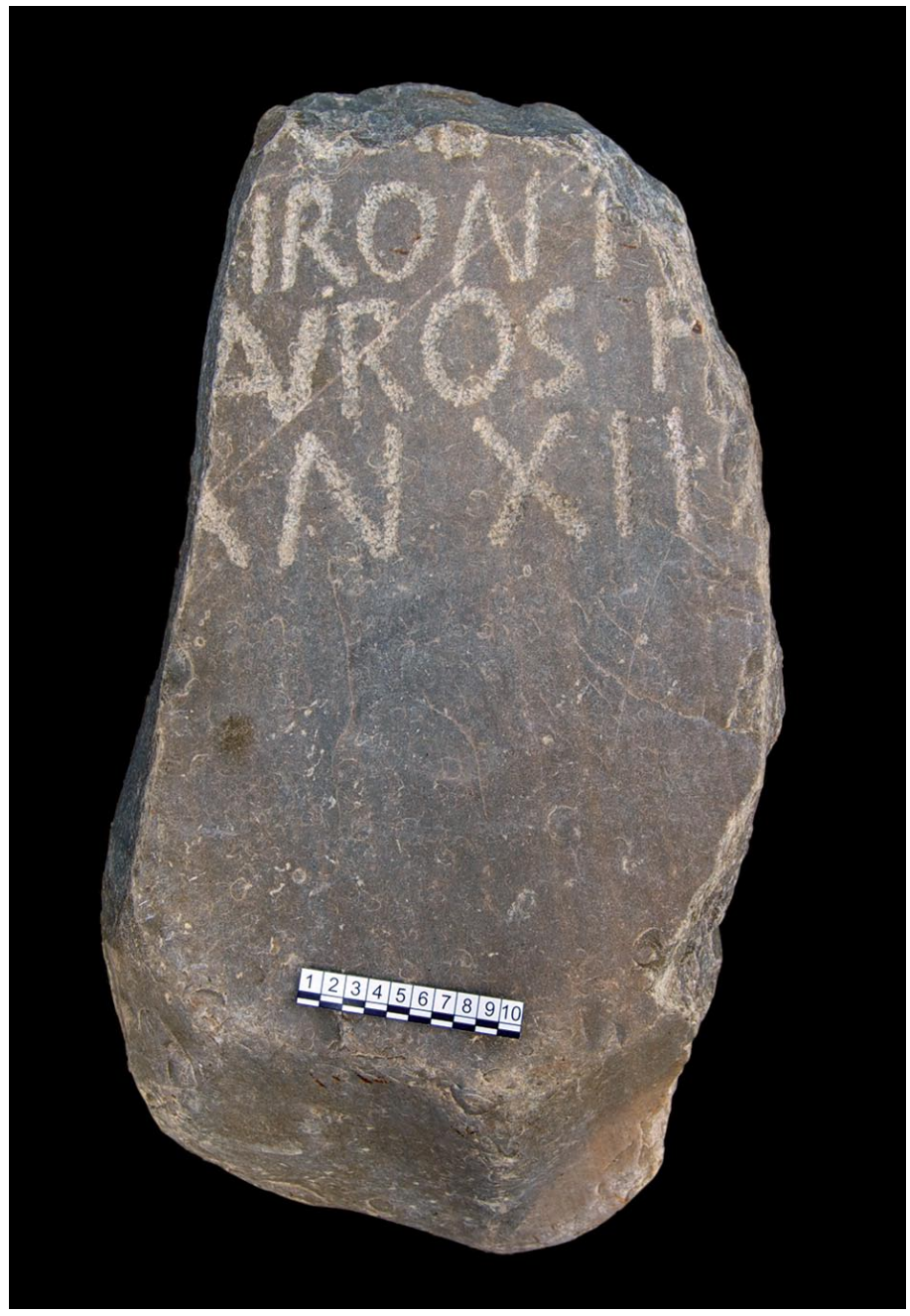

Figura I. Inscripción funeraria localizada en el río Tirón (Fresno de Rio Tirón, Burgos). Fotografía de los autores 
Transcripción:

$$
\begin{aligned}
& \text { ¿--- ? } \\
& \text { [-]IRONI } \\
& \text { [-]'AV'ROS.F } \\
& \text { AN XII }
\end{aligned}
$$

Interpretación:

$$
\text { ¿--- ? / V]ironi / [T]'au'ros ·f(ili) / an(norum) XII }
$$

Traducción:
(Monumento de) Virono, hijo de Tauro, de 12 años.

Estamos pues, ante una estela funeraria que sigue el esquema habitual de las estelas del conjunto de Belorado: nombre del difunto + filiación + edad del fallecido. En este caso el nombre del difunto lo encontramos en genitivo mientras que la terminación del nombre paterno presenta una serie de dificultades que comentaremos posteriormente. La descontextualización arqueológica de la pieza junto a la ausencia de los elementos habituales para establecer la datación de las inscripciones funerarias (fórmulas de consagración, adjetivos laudatorios, características paleográficas, etc.) ${ }^{9}$ nos impide poder especificar la datación de esta estela. Por lo tanto, solo podemos atribuirle una cronología amplia, comprendida entre los siglos i al III d.C., es decir, el periodo en el que se produjeron la mayoría de monumentos epigráficos en el Imperio Romano.

Respecto a los restos de repiqueteado de la parte superior del bloque fluvial, creemos que las posibilidades son dos: que se trate de una primera línea del texto en la que estaría el primer elemento del nombre del difunto o que sea uno de los signos que se encuentran en las inscripciones de este conjunto. Ambas opciones son posibles y presentan argumentos a favor y en contra.

Entre las razones que nos llevan a pensar en una primera línea que contendría el primer elemento del nombre del difunto, está el hecho de que el nombre doble sea el más habitual entre los difuntos representados en las inscripciones de este tipo. Tenemos varios ejemplos de tria y duo nomina pero solo uno en el que la difunta porta un único nombre ${ }^{10}$.

Por otro lado, respecto a la ordinatio del texto, se observa un mayor interlineado $(1,7 \mathrm{~cm})$ entre estos restos de repiqueteado y la primera línea identificable que entre las siguientes $(0,3-0,5 \mathrm{~cm})$. Esta mayor separación podría apuntar a que se tratase de un elemento externo al texto, sin embargo, la irregularidad en el interlineado no es extraña entre las inscripciones del lugar. Finalmente, los restos de repiqueteado no van desde el lado izquierdo hasta el final de la derecha sino que se interrumpen antes de llegar al final. Si fuese una palabra, deberíamos esperar encontrar más restos de repicado hasta el extremo derecho del bloque.

En resumen, hay argumentos que apuntan a ambas posibilidades pero no creemos que ninguna de ellas sea concluyente, debido a que el estado de conservación de la estela hace difícil decantarse de forma segura por una u otra.

La valoración onomástica de los restos de nombres que aparecen en la inscripción ofrece un panorama complejo. No cabe duda de que estereométricamente falta un solo carácter al inicio tanto

\footnotetext{
9 Sobre estas cuestiones generales, entre otros, se puede ver, para Hispania: Haley 1986, Knapp 1992, Stylow 1995 y Edmondson 2009 y para otras zonas del Imperio: Raepsaet-Charlier, Dondin-Payre 1999.
} 
de L. 2 como de L. 3. A partir de ahí cabe especular con la posible reconstrucción de los restos antroponímicos allí grabados.

En cuanto a la L.2, creemos que el antropónimo debería ser interpretado como el genitivo de Vironus, por tratarse del antropónimo del que más paralelos conocemos en Hispania (Vallejo Ruiz 2005, 455 $)^{11}$ incluidos algunos geográficamente cercanos como en la propia provincia de Burgos, en Hontoria de la Cantera, Auscus Boutius Vironi f. (ERLara, 14) y otro en Herramélluri (La Rioja) Segius Virono Matieni f. (ERR, 48).

Respecto a la L. 3, creemos que la reconstrucción más plausible del antropónimo sería la de Taurus (Vallejo Ruiz 2005, 424-425), por tratarse también del antropónimo más documentado en nuestro ámbito geográfico, contando incluso con un testimonio en Belorado que remite a la misma base: Quemia Taurometa Balbi f. (Hernando Sobrino 2009, 498-499) ${ }^{12}$.

Una cuestión de mayor complejidad es la presencia de la terminación -os donde cabría esperar un genitivo, ya que la estructura recurrente de los epitafios de Belorado, que consiste en el nombre del difunto seguido de su filiación y la edad a la que falleció, nos hace descartar que [T]auros esté funcionando como nominativo en un error $\langle\mathrm{us}=\mathrm{OS}>$. El análisis de las inscripciones de BeloradoFresno de Río Tirón en su conjunto, aconseja descartar una interpretación del tipo [T]aur $<u=O>s$ $f($ ilio) (posuit) en la que actuaría como padre del difunto y dedicante de la inscripción, ya que se trata de una estructura ajena a las más de 40 inscripciones que componen este grupo, es decir, se trataría de un unicum. En vez de ello, creemos que el antropónimo, independientemente de su terminación, es decir, de la forma que aquí presenta, podría estar haciendo referencia a la filiación paterna. La falta de concordancia entre la terminación y la función sintáctica de la palabra puede tratarse de un fenómeno puntual debido a diversas causas. Estas podrían ir desde una interferencia lingüística ${ }^{13}$ provocada por el mantenimiento de la antroponimia indígena ${ }^{14}$ hasta el mero error del lapicida, tal y como se constatan en algunas otras inscripciones latinas. Su análisis y explicación

11 Descartamos que se trate de Vironius, Tiro o Tironius por tratarse de antropónimos con mucha menor presencia que el mencionado.

12 Existe otra inscripción, también en paradero desconocido, [Q]uemiâe Iacometâe Ligir[i] / filia(e) anno(rum) $L X X$, muy similar y que podría dar pie a pensar que se trata de la misma pieza $(H E p 18,70)$. Sin embargo, y a pesar de que el primer idiónimo de la difunta es idéntico, filiación, abreviaturas, edad y disposición de las líneas son diferentes. No es el único caso en el que la similitud de dos inscripciones daría lugar a confusión e identificación entre ambas: baste por ejemplo mencionar el caso de los clunienses Luatro Frontonis filio y Tuatro Frontonis filio (Carcedo de Andrés, Pradales Ciprés 2015, n. ${ }^{\circ} 15$ y 16), documentados en Idanha-a-Velha. Idanha-a-Nova (Castelo Branco, Portugal). Por otra parte, el antropónimo Quemia, procedente de una base coim- (Vallejo Ruiz 2005, 288-289), tiene una buena representación bajo la forma Coemea en Lara de los Infantes (Burgos), donde como ocurriría en el caso beliforano, se documentan además algunos casos de individuos con nombres casi idénticos, como por ejemplo Coemeae Desicae y Coemea Dessicae (ERLara, n. 158 y 183). Por último, hay que mencionar que este antropónimo no es extraño en el entorno autrigón, más allá de los dos testimonios mencionados, sino que al contrario, es posible apreciar un ejemplo más en Belorado (Burgos), Quemia Maglaena (HEp 10, 91) y otro en Monasterio de Rodilla (Burgos), Quemia Boudica (Abásolo, Alonso, Sainz 1982, 164, n.o 4).

13 Gorrochategui ya expuso recientemente algunos interesantes ejemplos de distintas interferencias entre latín y celtibérico en la epigrafía (Gorrochategui 2011, 201-216).

14 Stüber menciona que la sustitución de los nombres galos por latinos no es un fenómeno irreversible, con casos de aparición de elementos lingüísticos indígenas que en algún caso parecen afectar efectivamente a la declinación dentro de un contexto netamente latino (Stüber 2007, 89, nota 7). Por otra parte, Adams, aunque para el caso del contacto entre latín y griego, menciona que es la expresión de la filiación el elemento más proclive a conservar una flexión indígena (Adams 2003, 376-380). Aun en dominios lingüísticos distintos - el contexto de la zona no invita a considerar la presencia de antropónimos helenos-, no está de más recordar que el elemento onomástico extraño del epígrafe de Fresno de Río Tirón correspondería precisamente a la filiación. 
general, en cualquier caso, excede el objeto de este estudio y deberá ser analizado y explicado por lingüistas ${ }^{15}$.

2. (Figs. 2 y 3) La segunda de las estelas fue descubierta el 24 de abril de 2015 con motivo de la limpieza de las orillas del río Retorto a su paso por el casco urbano por parte de un grupo de voluntarios $^{16}$. Actualmente se encuentra en la propiedad privada de su descubridor, Antonio Santamaría.

Se trata de un bloque fluvial de cuarcita, de morfología prismática irregular cuyas dimensiones son [65] $\times 37,5 \times 42 \mathrm{~cm}$. La pieza parece fracturada en su parte inferior, si bien esta fractura y las irregularidades de su forma, no afectan al texto conservado cuya lectura es clara.

La estela no está trabajada y carece de decoración a excepción a un signo que, como ya hemos comentado previamente, tiene paralelos semejantes en otras inscripciones de la zona, si bien desconocemos su significado ${ }^{17}$. En este caso, el signo tiene unas dimensiones de $6,8 \mathrm{~cm} \mathrm{y}$, como es habitual, se encuentra situado sobre la primera línea del texto, centrado. En cuanto a su forma, está formado por tres líneas rectas que recuerdan al perfil de una silla mirando hacia la izquierda.

El campo epigráfico se encuentra en la cara más lisa de la estela y mide $29 \times 25 \mathrm{~cm}$. Su estado de conservación es bueno aunque presenta algunas grietas en su superficie que no impiden su lectura y comprensión. El texto se encuentra alineado al lado izquierdo, guardando un margen de $5 \mathrm{~cm}$ en la primera línea que va disminuyendo debido a la forma del soporte. Está dividido en 5 líneas que tienen un interlineado irregular que oscila entre $0,5-2 \mathrm{~cm}$. El tamaño de las letras es igualmente irregular siendo la primera de las líneas la mayor con 4,5-4,7 cm, la segunda $2,8-3,1$, la tercera $2,5-3 \mathrm{~cm}$, la cuarta $3-4 \mathrm{~cm}$ y finalmente, la última línea $2,4-3,1 \mathrm{~cm}$.

En cuanto al tipo de letra, los caracteres son en general de tipo capital aunque algo irregulares y de tendencia alargada. Entre los rasgos paleográficos, cabe reseñar que la A de L. 2 y L. 3 carece de travesańo, mientras que en la L. 4 lo tiene ${ }^{18}$, por otro lado, la E de L. 1 es de doble trazo vertical. Se aprecia interpunción circular en L. 2, L. 3 y L. 4.

Transcripción:

SEGONTIO

ALONCO $\cdot$ AMB

ATI · FILIO · MIL

ITI $\cdot$ ANORVM

XXIX

Interpretación:

Segontio / Alonco · Amblati · filio · milliti a an<n>orum / XXIX

Traducción:

A Segontio Alonco, hijo de Ambato, soldado, de 29 años.

15 Contamos con dos testimonios de Viscunos en Clunia (Peñalba de Castro, Burgos) (CIL II, 2908 y 2910) a los que se añade un tercero en Feteira (Belver, Gaviāo, Portalegre, Portugal) $(A E 1984,470)$ y $[R]$ ettigeno[s---] [A?]isarros en otra inscripción cluniense más $(H E p$ 2, 140). En entornos extrapeninsulares, es posible encontrar algunos testimonios: Adginnos ( $A E$ 2000, 1090) en Hermagor (Carintia, Austria); Agriccos (CIL XIII, 5640), en Tilena (Poully-sur-Vinganne, Francia); Andos (CIL XIII, 247) en Lugdunum Conue- narum (Valcabrere, Francia); Iul(ius) Aturios (CIL XIII, 1089) en Mediolanum Santonum (Saintes, Francia), etc.

16 Agradecemos a Antonio Santamaría las facilidades ofrecidas para el estudio de la estela y toda la información proporcionada.

17 Ver nota 5.

18 La combinación de distintas formas de la letra A, sobre todo, en su travesaño, en una misma inscripción es un rasgo paleográfico común en este conjunto (Reyes Hernando, 2000, n. ${ }^{\circ}$ 2, 4, 7, 8, 9). 


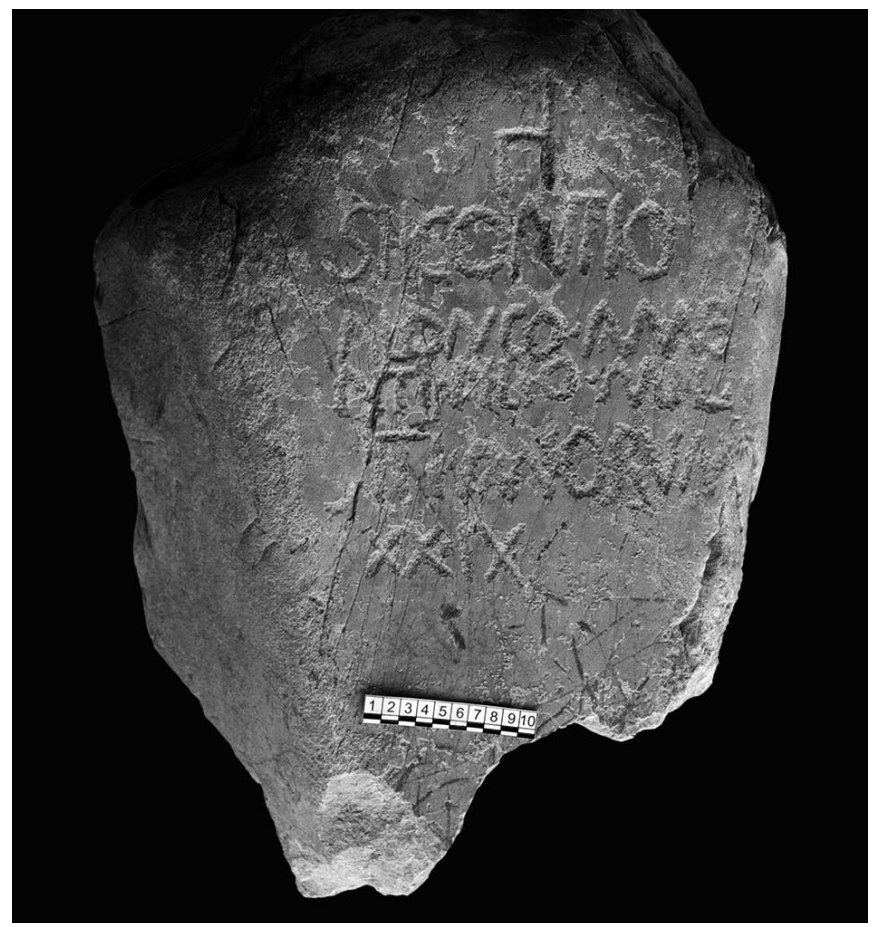

Figura 2. Inscripción funeraria localizada en el río Retorto (Fresno de Rio Tirón, Burgos). Fotografía de los autores

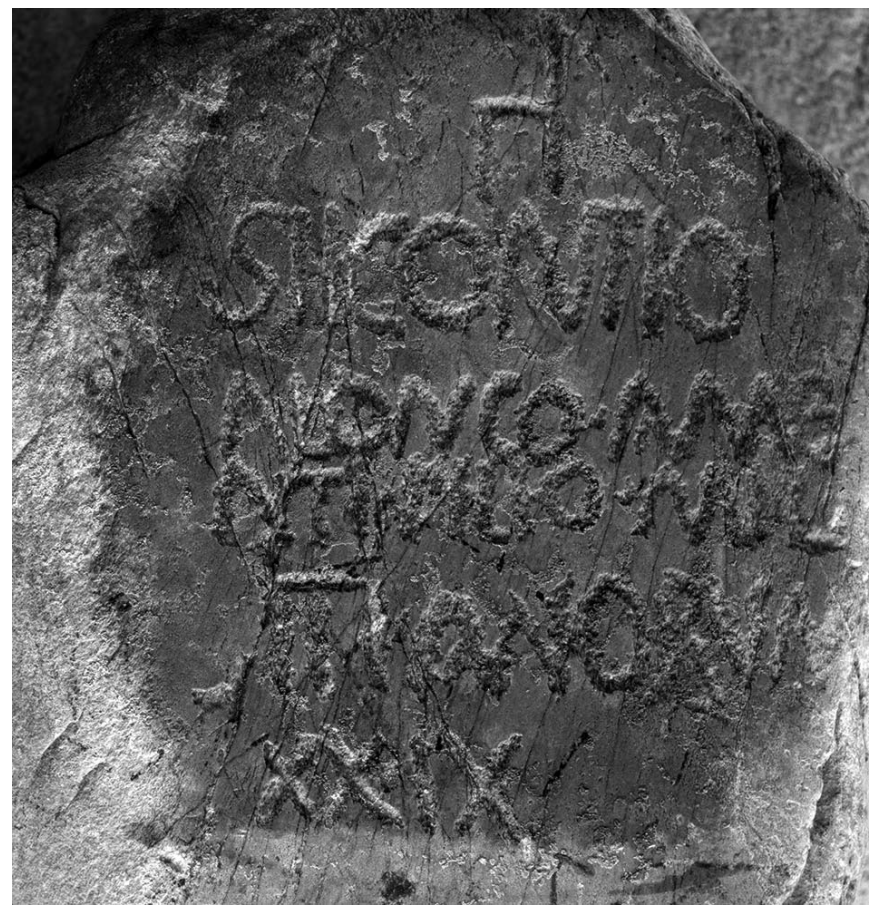

Figura 3. Detalle del campo epigráfico de la estela n. ${ }^{\circ} 2$ 
El texto muestra, una vez más, la estructura característica de las inscripciones funerarias de este lugar compuesta por el nombre del difunto — doble, en la mayoría de ocasiones - seguido de la filiación paterna y la edad a la que falleció. En este epígrafe, sin embargo, contamos con una novedad, la mención de la ocupación del difunto que señala su condición de soldado sin que podamos saber a qué unidad sirvió por falta de datos que nos lo indiquen ${ }^{19}$.

Como peculiaridad, cabe señalar que en este caso no se han utilizado las abreviaturas comunes para la palabra filio o annorum sino que se han escrito al completo. A pesar de que en este segundo caso, se escribió con una sola $n$, posiblemente, como consecuencia de un error del lapicida o de la ignorancia de la ortografía correcta.

Como en el caso de la anterior inscripción y la mayoría de las estelas del conjunto, nos es imposible concretar la cronología de la estela debido a la falta de contexto arqueológico u otros elementos como fórmulas funerarias que nos ayude en la tarea. En consecuencia, solo podemos señalar que su realización debió de tener lugar en algún momento entre los siglos I y III d. C, periodo de mayor expansión y producción epigráfica del Imperio romano ${ }^{20}$.

En cuanto a su onomástica, el difunto presenta una estructura típica de las estelas del lugar, es decir, un nombre indígena doble seguido de la filiación paterna. El primer elemento del nombre del difunto, Segontius, nos es de sobra conocido tanto en su versión sorda (Secontius), como en este caso, sonora (Abascal Palazón, 1994, 499 y 502 respectivamente). Sus paralelos se concentran sobre todo en Navarra y Araba/Álava con extensiones hacia La Rioja, Burgos y el territorio astur (Vallejo, 2005, 397).

En lo que respecta al segundo elemento del nombre del difunto, respondería a una de las numerosas formaciones sobre le radical $A l(l)$ - presentes en la antroponimia hispana (Vallejo, 2005, 118-121). Contamos con un paralelo claro en Villar del Rey (Badajoz), Accius Bouti f. Aloncus (AE 1971, 146) y una variante en Remolina (León) Tridio Alongun Bode(ri) f. (IRPL, $282=E R P L$, 391).

El nombre paterno, Ambatus, es el nombre indígena más veces atestiguado en la Península Ibérica (Abascal Palazón, 1994, 260) y es además, especialmente común en el espacio geográfico que nos ocupa ${ }^{21}$.

Contamos, por lo tanto, con una antroponimia de tipo indígena tanto en su estructura como carácter lingüístico que sigue los parámetros habituales del lugar donde lo más común es encontrar nombres indígenas dobles.

\section{Conclusión}

En conclusión, estos nuevos epitafios nos dan más información sobre el tipo de monumentos funerarios presentes en el área de Belorado-Fresno de Río Tirón (Burgos). Ambas estelas siguen de

\footnotetext{
19 Aunque lo más habitual es que la unidad militar este señalada, contamos con otro ejemplo cercano en el que tampoco se especifica en Zalduondo (Arabal Álava) ( $A E$ 1994, 1045), así como en Castrecias (Burgos) (IRPP, 101), y más alejado, en Arancón (Soria) (CIL II 2835) y en Ciutadella de Menorca (Islas Baleares) (CIL II 3716).

20 Ver nota 9.
}

21 En Fresno de Río Tirón Metelius Reburrus Ambatif. y en Belorado Ambato Alebbio Bodani f., Ambata Medica Verati f., Segilus Aespancus Ambata f., Ambatus Burga Segilif. y Ambata Peditaga Ambati f. (Reyes Hernando 2000, n. ${ }^{\circ}$ 5, 9, 20, 21 y 24 respectivamente), [Am]bata [Se]gon[ti]a (Gorrochategui y Fernández Corral, e.p., n.o 4) y variantes como Ambaia Doghica Vigani f. y [Ca]s(sia) Ambade Festi f. (Fernández Corral, 2015, n.o 1). 
modo general las características propias de la epigrafía de este lugar — que ya hemos adelantado al comienzo del trabajo-, unas singularidades que han permitido identificarlas como un conjunto bien definido y que hace necesario que sus ejemplares sean estudiados siempre dentro de su contexto.

Si bien la primera estela analizada presenta algunas complicaciones debido a su estado de conservación y lo particular de la forma de la filiación del difunto, la segunda tiene una lectura mucho más clara. Además, nos aporta un nuevo elemento como es la mención de un difunto que había formado parte de alguna unidad militar que no se especifica. La falta de algún detalle más que la palabra miles y la ausencia de elementos que nos indiquen la cronología de la estela, hacen difícil plantear hipótesis sobre la unidad militar sirvió el difunto.

Por otro lado, la aparición de esta estela con la palabra miles desarrollada, nos hace replantearnos la interpretación de otra estela aparecida también en Fresno de Río Tirón en la que se podría estar mencionando también a un militar. En efecto, la interpretación de la estela dedicada a Horatius Alionus Ligiri f. sembraba dudas por la presencia de una $\mathrm{M}$ situada tras el nombre del difunto que se ha identificado con la abreviatura de la palabra $m$ (onumentum), m(erensis) o $m$ (emoriam ${ }^{22}$. No obstante, el descubrimiento de la última estela en Fresno de Río Tirón nos presenta una nueva posibilidad de interpretación de la letra $\mathrm{M}$ como abreviatura de $m$ (iles). Lamentablemente, al igual que con las propuestas anteriores, es imposible decantarnos con total seguridad por esta opción u otra.

Si bien la peculiaridad del soporte de los epitafios de este lugar es evidente, aunque no un unicum $^{23}$, no queremos dejar de señalar que el análisis de las inscripciones apuntan claramente a los estándares epigráficos romanos, con la presencia de abreviaturas comunes como $f$ (ilius) o an (norum $)^{24}$, nexos en las letras, interpunciones, etc. Por lo tanto, creemos que la elección de estos soportes no responde ni a una limitación material o técnica ni a una influencia de la cultura epigráfica romana débil, sino a una elección consciente de la población local que podría estar relacionada con cuestiones sociales, culturales o religiosas que, por el momento, con los datos disponibles resultan difíciles de conocer.

\section{BiBLIOGRAFÍA}

Abascal Palazón, J. M., 1994, Los nombres personales en las inscripciones latinas de Hispania, Murcia: Universidad de Murcia, Universidad Complutense de Madrid y Universidad de Alicante.

AвÁsolo , J. A., 1974, Carta arqueológica de la provincia de Burgos I: partidos judiciales de Belorado y Miranda de Ebro, Santiago de Compostela-Valladolid: Universidad de Santiago de Compostela.

Abásolo, J. A., Alonso, J. M., Sainz, F., 1982, «Nuevas inscripciones romanas procedentes de Briongos y Monasterio de Rodilla», en Boletín de la Institución Fernán González 198, 161-168. (http://riubu.ubu.es/ bitstream/10259.4/2065/1/0211-8998_n198_p161-168.pdf)

Adams. J. N., 2003, Bilingualism and the Latin Language, Cambridge: Cambridge University Press.

Carcedo de Andrés, B. P., Pradales Ciprés, D., 2015, Clunienses fuera de Clunia. Un estudio epigráfico, [Colección Fernán González 5], Burgos: Real Academia Burgense de Historia y Bellas Artes Institución Fernán González.

Delamarre, X., 2007, Noms de personnes celtiques dans l'épigraphie classique, Paris: Errance.

22 Encontramos estas dudas expresadas en Reyes

Hernando 2000, n. ${ }^{\circ} 12$.

23 Ver nota 2.
24 La segunda estela presentada en este trabajo representa una excepción en este sentido. 
Dondin-Payre, M. y Raepsaet-Charlier, M.-Th., 1999, "Critères de datation épigraphique pour les Gaules et les Germanies», en: M. Dondin-Payre y M.-Th. Raepsaet-Charlier (eds.), Cités, municipes, colonies: les processus de municipalisation en Gaule et en Germanie sous le Haut Empire romain, VII-XII, Paris: Publications de la Sorbonne.

Edmondson, J., 2001, «Datación de los monumentos: criterios paleográficos», en: J. Edmondson, T. Nogales, y W. Trillmich (eds.), Imagen y memoria. Monumentos funerarios con retratos en la colonia Augusta Emerita, 63-04, Mérida: Real Academia de la Historia-Museo Nacional de Arte Romano.

Fernández Corral, M., 2015, «Nuevos monumentos funerarios en Belorado (Burgos)», Lucentum 34 [en prensa].

GonzÁlez Rodríguez, M. ${ }^{a}$ C., 1997. Los astures y los cántabros vadinienses: problemas y perspectivas de análisis de las sociedades indígenas de la Hispania indoeuropea, [Anejos de Veleia. Series minor 10], VitoriaGasteiz: Universidad del País Vasco/Euskal Herriko Unibertsitatea.

—,2005, "Epigrafía y sociedad: el ejemplo vadiniense», en: Solórzano Telechea, J.A., González Morales, M.R. (eds.), II Encuentro de Historia de Cantabria: actas del II Encuentro celebrado en Santander los dias 25 a 29 de noviembre del año 2002, Tomo I, Santander: Universidad de Cantabria, 91-115.

Gorrochategui, J., 2011, "Interferencias lingüísticas en el material epigráfico hispano-celta», en: Luján, E.R., García Alonso, J. L. (eds.), A Greek Man in the Iberian Street. Papers in Linguistics and Epigraphy in Honour of Javier de Hoz, [Innsbrucker Beiträge zur Sprachwissenschaft 140], Innsbruck: Institut für Sprachen und Literaturen der Universität Innsbruck, 201-216.

Gorrochategui, J. y Fernández Corral, M., e.p., «Cinco nuevas estelas funerarias procedentes de Belorado (Burgos)", Zeitschrift für Papyrologie und Epigraphik.

Haley, E. W., 1986, Foreigners in Roman Imperial Spain: Investigations of Geographical Mobility in the Spanish Provinces of the Roman Empire 30 B.C.-A.D. 284, New York: Ph. Diss. University of Columbia.

Hernando Sobrino, M. R., 2009, Manuscritos de contenido epigráfico de la Biblioteca Nacional de Madrid (siglos XVI-XX). La transmisión de las inscripciones de la Hispania romana y visigoda, [Colección Libros de Investigación], Madrid: Centro de Lingüística Aplicada Atenea.

Knapp, R. C., 1992, Latin Inscriptions from Central Spain. California: University of California Press.

Oller, J., De León, D., Eguiluz, D., 2015, Informe de la intervención arqueológica en los yacimientos de La Muela, La Mesa y El Castillo. Campaña 2014. (Informe inédito).

Reyes Hernando, O.V., 2000, El Conjunto Epigráfico de Belorado. Valladolid: Universidad de Valladolid.

STÜBER, K., 2007, «Effects on language contact on Roman and Gaulish Personal Names», en: Tristram, H.L.C. (ed.), The celtic languages in contact. Papers from the workshop within the framework of the XIII International Congress of Celtic Studies, Bonn, 26-27 July, 2007, Potsdam: Potsdam University Press, 81-92. (https://publishup.uni-potsdam.de/opus4-ubp/frontdoor/index/index/docId/691)

STYlow, A., 1995, "Los inicios de la epigrafia latina en la Bética. El ejemplo de la epigrafía funeraria», en: F. Beltrán Lloris (ed.), Roma y el nacimiento de la cultura epigráfica en Occidente, 219-238. Zaragoza: Institución Fernando el Católico.

Vallejo Ruiz, J. M., 2005, Antroponimia Indigena de la Lusitania Romana, [Anejos de Veleia. Series minor 23], Vitoria-Gasteiz: Universidad del País Vasco/Euskal Herriko Unibertsitatea.

\section{Abreviaturas}

$A E=$ L'Année Épigraphique, París, 1988 ss.

$C I L=$ Corpus Inscriptionum Latinarum, Berlín, 1863 ss.

ERLara = AвÁsolo, J.A., 1975, Epigrafía romana de la región de Lara de los Infantes, Burgos: Diputación Provincial de Burgos. 
ERPL = Rabanal Alonso, M.A., García Martínez, S.M.a, 2001, Epigrafía romana de la provincia de León. Revisión y actualización, León: Universidad de León.

$E R R$ = Espinosa, U., 1986, Epigrafía Romana de La Rioja, Logroño: Instituto de Estudios Riojanos.

$I R P L=$ Diego SAntos, F., 1986, Inscripciones romanas de la provincia de León, León: Institución Bernardino de Sahagún, Diputación Provincial de León.

IRPP = Hernández Guerra, L., 1994, Inscripciones romanas en la provincia de Palencia, Valladolid: Universidad de Valladolid. 